\title{
A Target for Rosetta
}

\author{
Rita Schulz \\ Research and Scientific Support Department of ESA, ESTEC, Postbus \\ 299, 2200 AG Noordwijk, The Netherlands
}

\begin{abstract}
Comet 67P/Churyumov-Gerasimenko has been chosen as the new target of the Rosetta mission. A concise overview of the basic properties of the new target comet is given.
\end{abstract}

\section{Introduction}

ESA's Rosetta mission is the first comet mission that will not only fly-by a comet nucleus. Rosetta will go in orbit around the nucleus to investigate its evolution along a major part of its orbit and land on it to obtain in-situ information of the surface and subsurface composition and physical properties. It was originally scheduled to launch to Comet $46 \mathrm{P} /$ Wirtanen in January 2003. However, as the launch had to be delayed owing to unforeseen problems with the launch vehicle, the original launch window closed and alternative mission opportunities had to be found. Comet $67 \mathrm{P} /$ Churyumov-Gerasimenko has now been identified as the new Rosetta target and the mission is scheduled for launch in February 2004. However, before this decision could be taken, a number of basic properties of the new target comet had to be determined to ensure that the rendezvous and landing on the nucleus will be successful. The design of the scientific instruments on board the Orbiter as well as the design of the landing scenario had been optimized to the characteristics of $46 \mathrm{P} /$ Wirtanen, as determined from ground. Consequently, the properties of the new target comet, particularly its size and activity had to be within the range required to guarantee the feasibility of the mission and an optimal scientific return. Comet 67P/Churyumov-Gerasimenko was therefore extensively observed between February and June 2003. The major results of these observations will be summarized here.

\section{Monitoring Comet 67P/Churyumov-Gerasimenko}

A huge amount of observations of Comet $67 \mathrm{P} /$ Churyumov-Gerasimenko was obtained in 2003, after it was announced to be the likely new target for Rosetta. The most complete monitoring was conducted at the European Southern Observatory, where the comet was observed on 11 nights between 11 February and 26 June 2003. Broad-band BVR images and low-resolution long-slit spectra were obtained at the ESO Very Large Telescope as well as the $3.6 \mathrm{~m}$ Telescope for morphological, colour and compositional analysis of the coma and for studying the evolution of the comet's activity along the postperihelion orbit between 2.29 $\mathrm{AU}$ and 3.22 AU. In May 2003 the observational strategy was specifically ad- 
justed to obtain data suitable for the analysis of the rotational properties of the nucleus. A summary of the observations is given in Table 1.

Table 1. Postperihelion Monitoring from ESO in 2003

\begin{tabular}{c|c|c|c} 
Date & Telescope & Type of Observation & $\mathrm{r}_{h}[\mathrm{AU}]$ \\
\hline 10/11 Feb & $3.6 \mathrm{~m}$ & BVR imaging + spectroscopy & 2.29 \\
8/9 Mar & $3.6 \mathrm{~m}$ & BVR imaging + spectroscopy & 2.49 \\
30 Apr - 4 May & VLT & BVR imaging + spectroscopy & $2.85-2.88$ \\
3-4 June & VLT & BVR imaging & 3.08 \\
19-20 June & VLT & BVR imaging & 3.18 \\
23-26 June & VLT & BVR imaging & $3.21-3.22$
\end{tabular}

\section{Characteristics of Major Relevance}

Although they have not yet been completely evaluated the observations obtained in 2003 already resulted in a number of new findings that were vital to determine the basic characteristics of Comet $67 \mathrm{P} /$ Churyumov-Gerasimenko. Those most important in view to the selection of this comet as the new target for the Rosetta mission are briefly summarized. 67P/Churyumov-Gerasimenko showed a major drop of activity between $2.5 \mathrm{AU}$ and $2.9 \mathrm{AU}$. The dust production rate (in $A f \rho$ ) decreased by more than a factor of two and the CN production rate decreased by about one third. This confirms that although the comet is more active than $46 \mathrm{P} /$ Wirtanen when closer to the sun (i.e. its dust production at $2.3 \mathrm{AU}$ is about 1.5 times that of $46 \mathrm{P} /$ Wirtanen) the activity of both comets is very similar at around $3 \mathrm{AU}$, which is the heliocentric distance at which the Lander will be deployed onto the nucleus surface. The effective radius of the comet nucleus was determined to be $1.98 \pm 0.02 \mathrm{~km}$ from HST observations obtained in March 2003 (Lamy et al., 2003). With the above information it could be confirmed that a safe landing is possible also on the nucleus of Comet $67 \mathrm{P} /$ ChuryumovGerasimenko. Lamy et al. (2003) inferred a rotation period of $12.3 \pm 0.27$ hours on the basis of the HST observations obtained in a time interval of 12 hours. The detailed analysis of the ground based monitoring data that have been dedicated to the determination of the rotational properties is still under way. The comet displayed various distinct features in the coma as well as an anti-tail which can be used to determine, or at least constrain, some basic properties of the nucleus, such as its rotational properties and the number and location of active regions on the nucleus' surface.

\section{Conclusions}

Comet $67 \mathrm{P} /$ Churyumov-Gerasimenko is not only a suitable target for the Rosetta mission, but actually a very interesting one. In contrast to $46 \mathrm{P} /$ Wirtanen, this comet exhibits distinct coma features (jet structures) similar to those of Comets $1 \mathrm{P} /$ Halley and $19 \mathrm{P}$ /Borrelly, the only two comets for which images of the nucleus exist. Hence, Rosetta will be able to proof whether such structures are 
indeed a direct result of the nucleus being divided in active and inactive regions. Observations during previous apparition have already shown that Comet $67 \mathrm{P} / \mathrm{Churyumov-Gerasimenko} \mathrm{shows} \mathrm{a} \mathrm{seasonal} \mathrm{effect} \mathrm{when} \mathrm{it} \mathrm{passes} \mathrm{its} \mathrm{perihe-}$ lion. Such an effect has generally been attributed to a new active region being exposed to sunlight. The new mission scenario of Rosetta foresees a monitoring of its target not only during its preperihelion phase, but through perihelion passage and along a significant part of its postperihelion orbit. Therefore, Rosetta will not only be able to study the development of cometary activity at large distances, but also very close to the sun, i.e., when the seasonal effect occurs.

Acknowledgments. A huge amount of support was received by individual scientists and observatories in the effort to define the new target comet of the Rosetta mission. ESO and HST granted Directors Discretionary Time on short notice, which is gratefully acknowledged.

\section{References}

Lamy, P. L., Toth, I., Weaver, H., Jorda, L., \& Kaasalainen, M. 2003, BAAS, 35, 970 